\title{
Modelling the hysteretic wale-wise stretching behaviour of technical plain knits
}

\author{
Gilles Dusserre* \\ Université de Toulouse, Mines Albi, ICA (Institut Clément Ader), Campus Jarlard, F-81013, Albi, France
}

\begin{abstract}
A B S T R A C T
This paper investigates the effect of friction on the stretching behaviour of plain-knits in the wale-wise direction. The capstan method was carried out to measure the yarn-to-yarn coefficient of kinetic friction between perpendicular yarns. The coefficient of kinetic friction between E-glass yarns and basalt yarns is similar and exhibits significant normal load sensitivity. However only E-glass yarns exhibit sensitivity to the sliding velocity. The yarn-to-yarn friction was then introduced in an existing semi-analytical model to simulate the wale-wise stretching of plain knits. This model was used to perform a sensitivity study, which has shown that friction significantly affects the knit behaviour both before and after jamming. The enhanced model proved to be able to properly describe the hysteretic behaviour of plain knits in the wale-wise direction. This allows concluding that friction at the loops crossing point is the main mechanism that controls the hysteretic behaviour of plain-knits, even if other mechanisms should be accounted for to perfectly describe the actual behaviour of the knit.
\end{abstract}

\section{Introduction}

Increasing the production rate of complex parts is among the main issues currently investigated to develop the use of composite materials in mass production industries. Liquid Composite Moulding techniques are promising ways to match these requirements to process thermoset resins. These processes require forming the reinforcement while or before placing it in the mould and permeate it with a low viscosity resin. Textile composites are thus widely investigated because they comprise several fibre orientations in a single ply and allow an efficient preforming step. Forming modelling (Allaoui et al., 2011) is an important issue to predict the fibres orientation in the preform and thus control the local permeability of the reinforcement (Heardman et al., 2001; Dusserre et al., 2011) and the final properties of the composite material as well (Truong et al., 2008; Dusserre et al., 2014). Knit reinforced composites belong to the family of textile composites and present many advantages for the manufacturing of complex shape preforms: 3D net-shape preforms, possibly holed, can be knitted automatically without cutting the yarns, with several types

\footnotetext{
* Tel.: +33563493309.

E-mail address: gilles.dusserre@mines-albi.fr.
}

of fibre, various local knitting patterns and thus various local thicknesses and properties (Leong et al., 2000; Padaki et al., 2006; Balea et al., 2014; Dusserre and Bernhart, 2015). Forming a knit involves a change in the stitch geometry resulting from shearing and stretching, both mainly controlled by a yarn bending mechanism (Duhovic and Bhattacharyya, 2006).

A characteristic of knits is their stretch ability (Senthilkumar and Anbumani, 2011). Two steps mainly characterize the tensile behaviour of technical knits in wale-wise stretching. The first one, before jamming, involves high deformation under low load. In this step the sliding at the loops crossing point plays an important role, especially while stretching the knit in the wale-wise direction. Popper (1966) assessed the role of friction in the biaxial behaviour of knitted fabrics through a theoretical analysis and demonstrated that its effect is very important even if the value of the coefficient of kinetic friction is low (0.1). This effect being opposed to the sliding direction, its contribution acts inversely during loading and unloading steps, leading to a very pronounced hysteretic behaviour of knitted fabrics under biaxial (Popper, 1966) and uniaxial (Matsuo and Yamada, 2009) tension. The sensitivity to friction is exacerbated by the exponential form of the drive belt formula that may apply at the loops crossing points due to yarn curvature (Popper, 1966).

The friction at the contact between two yarns depends on the relative orientation of both yarns. Indeed, the area in contact is 


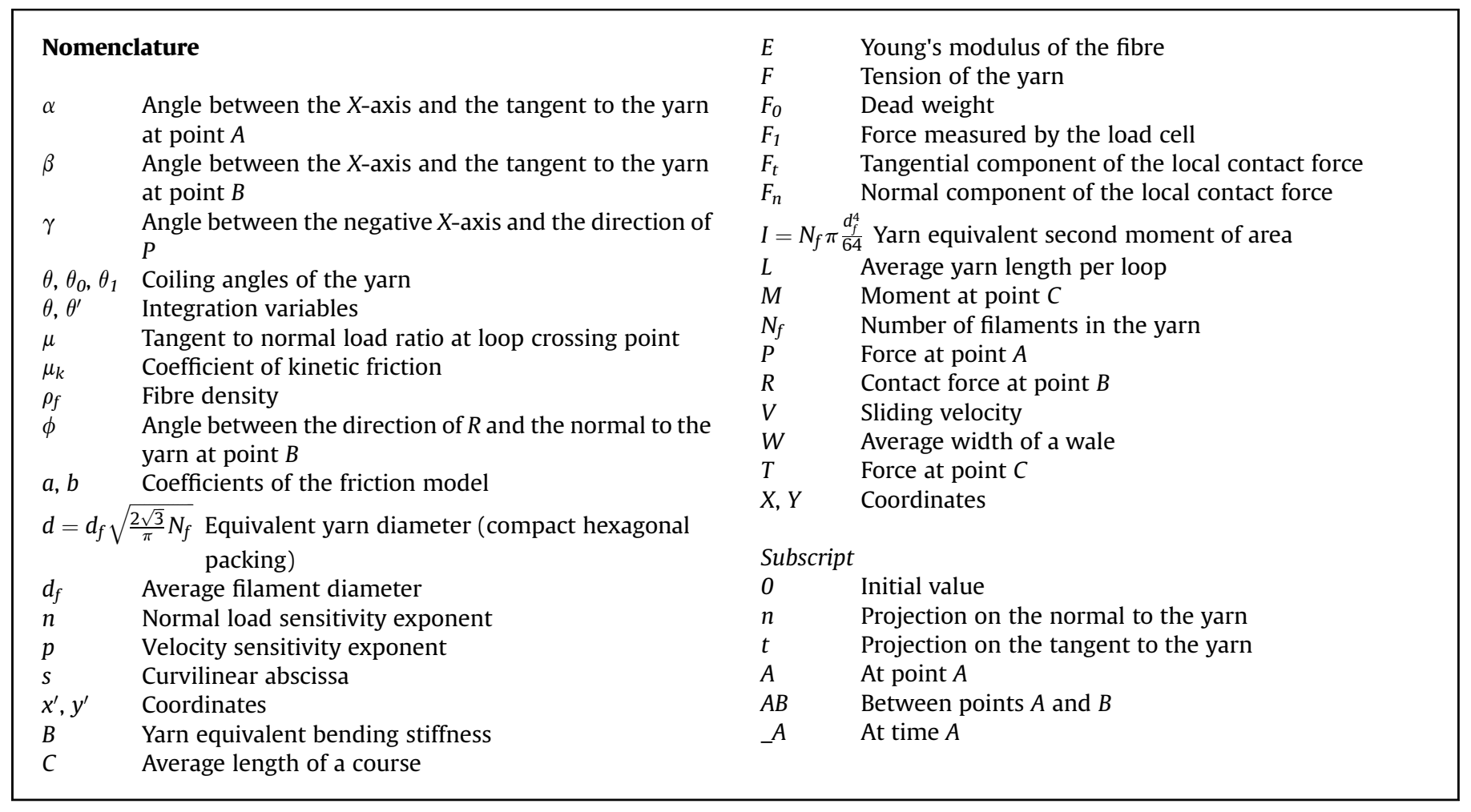

higher in the case of two parallel yarns than in the case of perpendicular yarns because the contact is linear instead of being punctual (Cornelissen et al., 2013). Moreover the coefficient of friction between two parallel yarns also depends on the sliding direction. The friction behaviour between two yarns is a macroscale description that embraces numerous micro-scale characteristics of the fibres and yarns and nano-scale phenomena (Behary et al., 2000). The value of the coefficient of friction can be experimentally assessed through several methods: twisted strand (Cornelissen et al., 2013), capstan (Cornelissen et al., 2013), Atomic Force Microscopy and Lateral Force Microscopy (Behary et al., 2000).

The present study contributes to understand the behaviour of dry knitted preforms by investigating the effect of friction on the behaviour of both E-glass and basalt knits. No value concerning the coefficient of friction between basalt fibres are reported in the literature. The values reported for E-glass fibres range between 0.4 and 1 for parallel contact depending on experimental method (Cornelissen et al., 2013). For perpendicular contact, values between 0.12 and 0.2 have been reported (Behary et al., 2000) depending on the sizing of the fibre. It was thus chosen to perform a measurement of the coefficient of kinetic friction related to the actual fibres of the knit studied.

This paper is devoted to extend the capabilities of an existing semi-analytical model by accounting for friction to simulate the hysteretic behaviour of E-glass and basalt plain knits subjected to wale-wise stretching. The original model (Hong et al., 2002) is based on a semi-analytical analysis of the momentum balance of a quarter stitch. It is proposed in this paper to introduce the angle of friction at the loops crossing point to perform an improved analysis. The actual contribution of friction at this point is discussed and the results of the model are analysed through a sensitivity study and a comparison to experiment. Despite a simplified description of the frictional behaviour, the improved model is able to reproduce properly the hysteretic behaviour of knits subjected to wale-wise stretching.

\section{Materials and methods}

\subsection{Fibres}

This paper investigates the effect of yarn-to-yarn friction on the tensile behaviour of fabrics knitted with E-glass and basalt fibres. The principal characteristics of the yarns are reported in Table 1. Both yarns are very similar and their characteristics were chosen to provide the same equivalent yarn diameter in order to knit fabrics with similar loop geometry. The main difference between both yarns is the lower bending stiffness of basalt yarns due to a lower average filament diameter despite a slightly higher Young's modulus.

\subsection{Knitted fabrics}

Plain knits were manufactured with these yarns on an industrial two-needle beds 5-gauge flat knitting machine. Fig. 1 schematizes the plain knit structure comprising only front stitches. In the $100 \mathrm{~mm} \times 300 \mathrm{~mm}$ specimens tested in this study, the average width of each of the 23 wales, $W$, is $4.4 \mathrm{~mm}$, the average length of each of the 110 courses, $C$, is $2.7 \mathrm{~mm}$ irrespective of the fibre type, and the average yarn length per stitch, $L$, is $14.1 \mathrm{~mm}$ for E-glass knits and $13.1 \mathrm{~mm}$ for basalt knits due to the higher bending stiffness of the former.

Table 1

Characteristics of the yarns (from manufacturers datasheets).

\begin{tabular}{lll}
\hline Yarn & E-glass & Basalt \\
\hline Fibres & Owens Corning Vetrotex T30 111AX23 & Basaltex BCF \\
$N_{f}$ & 1200 & 1672 \\
$d_{f}(\mu \mathrm{m})$ & 16 & 13 \\
$\rho_{f}\left(\mathrm{~kg} \mathrm{~m}^{-3}\right)$ & 2620 & 2700 \\
$E(\mathrm{GPa})$ & 80 & 84 \\
$d(\mathrm{~mm})$ & 0.58 & 0.56 \\
Linear density (tex) & 600 & 600 \\
$B=E I\left(\mathrm{Nm}^{2}\right)$ & 0.31 & 0.20 \\
Sizing & Silane based & Silane based \\
\hline
\end{tabular}




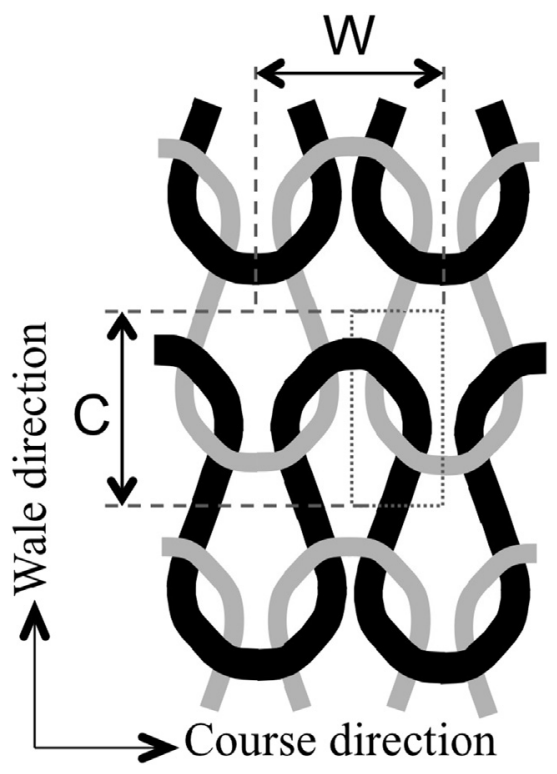

Fig. 1. Schematic diagram of a plain knit.

\subsection{Tensile tests}

Monotonic and cyclic tensile tests were carried out in the walewise direction at a constant crosshead displacement rate of $50 \mathrm{~mm} \mathrm{~min}^{-1}$ on an INSTRON 5800R electromechanical testing machine fitted out with a $30 \mathrm{kN}$-load cell. At each extremity of the specimen, $50 \mathrm{~mm}$ of knit were clamped into pneumatic grips fitted out with a rubber lining. The testing area is thus $200 \mathrm{~mm}$ long. The cyclic tests were performed with three cycles comprising a loading step up to a change in length of $60 \%$, followed by an unloading step up to a change in length of $10 \%$.

\subsection{Friction factor measurement}

A variation of the capstan method (Blau, 2008) was carried out to measure the coefficient of kinetic friction between two perpendicular yarns. The method chosen consists in measuring the load at each extremity of a wire that slides on two cylinders, as explained in Fig. 2. Two $10 \mathrm{~mm}$-diameter cylinders, radially drilled, were covered with 16 coils of a yarn parallel to their axis, allowing a whole covering of the cylinder surface (cf. inset in Fig. 2). The cylinders are then placed horizontally on an INSTRON 5800R electromechanical testing machine fitted out with a $500 \mathrm{~N}$-load cell. The load $F_{0}$ was applied at the lower extremity of the yarn thanks to a dead weight, whereas the upper extremity was linked to the load cell in order to measure the load $F_{1}$ during both ascending and descending steps of the crosshead.

$F_{1}$ and $F_{0}$ are related by the drive belt formula, equation (1), where $\theta_{1}$ and $\theta_{0}$ are the coiling angles corresponding to each cylinder. The cylinders were located in such a way that $\theta_{1}=\theta_{0}=\pi / 2$, allowing to assess the coefficient of kinetic friction $\mu_{k}$.

$\frac{F_{1}}{F_{0}}=\exp \left(\mu_{k}\left(\theta_{1}+\theta_{2}\right)\right)$

When the crosshead goes up, the load $F_{1}(10 \mathrm{~N})$ is higher than $F_{0}$ $(6.31 \mathrm{~N})$ due to friction effect, equation (1). When the crosshead goes down, the roles of $F_{1}$ and $F_{0}$ in equation (1) are inverted (the sign of the coiling angles is negative) and $F_{1}$ is lower ( $4 \mathrm{~N}$ ). It is noteworthy that the coefficient of static friction factor measured by this method is similar to the coefficient of kinetic friction since no load peak occurred just before the sliding, irrespective of the testing conditions. The values of the coefficient of kinetic friction determined through equation (1) considering the average values of $F_{1}$ during the ascent and descent of the crosshead are reported in Table 2 for sliding velocities ranging between 0.2 and $10 \mathrm{~mm} \mathrm{~s}^{-1}$ and for a dead weight of 6.31 and $12.62 \mathrm{~N}$. The coefficient of kinetic friction ranges between 0.14 and 0.18 irrespective of the fibre type. This is consistent with the values reported in Behary et al. (2000) for E-glass fibres, between 0.12 and 0.2 depending on the size. It is noteworthy that the value of $F_{1}$ is sufficiently stable on each identification range to consider the average value. The maximal standard derivation is 0.13 for a sliding velocity of $10 \mathrm{~mm} \mathrm{~s}^{-1}$, with an average value of $F_{1}$ respectively of $10.7 \mathrm{~N}$ and $9.94 \mathrm{~N}$ for E-glass and basalt fibres $\left(F_{0}=6.31 \mathrm{~N}\right)$.

The experimental results processed using equation (1) show that the coefficient of kinetic friction decreases when the load $F_{1}$ increases (increase of $F_{0}$ or ascent compared to descent) for both basalt and E-Glass yarns. Moreover, the coefficient of kinetic friction obtained with E-Glass fibres exhibits a slight dependence to the sliding velocity whereas this trend is not emphasised for basalt

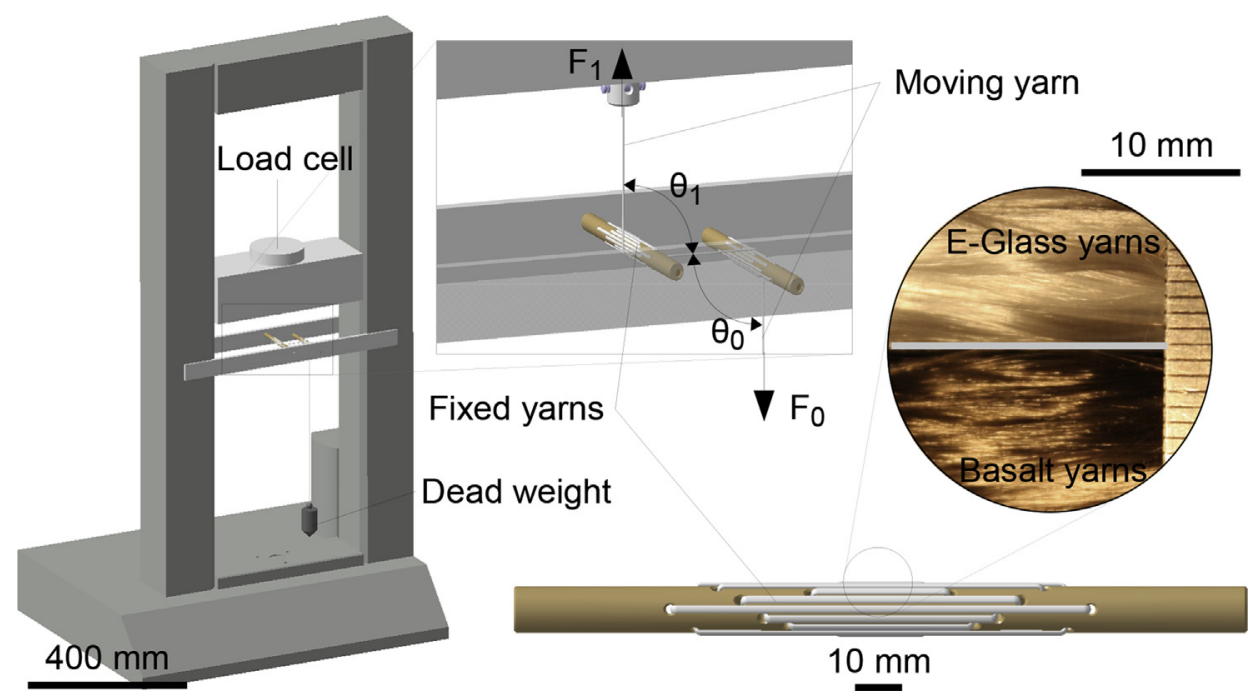

Fig. 2. Setup used to carry out the capstan method. 
Table 2

Coefficient of kinetic friction determined through equation (1).

\begin{tabular}{|c|c|c|c|c|c|c|c|}
\hline \multirow{2}{*}{\multicolumn{2}{|c|}{$\begin{array}{l}\text { Sliding velocity } \mathrm{V} \\
\left(\mathrm{mm} \mathrm{s}^{-1}\right)\end{array}$}} & \multicolumn{3}{|c|}{ E-Glass fibres } & \multicolumn{3}{|c|}{ Basalt fibres } \\
\hline & & 0.2 & 1 & 10 & 0.2 & 1 & 10 \\
\hline \multirow{2}{*}{$F_{0}=6.31 \mathrm{~N}$} & Ascent & 0.156 & 0.162 & 0.172 & 0.144 & 0.144 & 0.145 \\
\hline & Descent & 0.167 & 0.174 & 0.181 & 0.152 & 0.155 & 0.148 \\
\hline \multirow[t]{2}{*}{$F_{0}=12.62 \mathrm{~N}$} & Ascent & 0.152 & $\mathrm{~N} / \mathrm{A}$ & $\mathrm{N} / \mathrm{A}$ & $\mathrm{N} / \mathrm{A}$ & $\mathrm{N} / \mathrm{A}$ & $\mathrm{N} / \mathrm{A}$ \\
\hline & Descent & 0.157 & $\mathrm{~N} / \mathrm{A}$ & $\mathrm{N} / \mathrm{A}$ & $\mathrm{N} / \mathrm{A}$ & $\mathrm{N} / \mathrm{A}$ & N/A \\
\hline
\end{tabular}

fibres. Even if both technical datasheets (Table 1) mention a silane based sizing agent, this matter of fact may result from a difference in the sizing process (Behary et al., 2000; Baltina et al., 2007).

As verified by many authors (Gupta, 1992), the coefficient of friction between fibres depends on the normal force at the contact between both yarns. This dependence can be described by the Howell's equation (Howell and Mazur, 1953), equation (2), a power law that relates the tangential, $F_{t}$, to the normal, $F_{n}$, component of the reaction load.

$F_{t}=a F_{n}^{n}$

Introducing equation (2) in the well-known reasoning that leads to the drive belt formula (equation (1)) provides the differential equation (3), that relates the tension in the yarn, $F$, at an angular position $\theta$ and its variation, $d F$, along an angular element $d \theta$. For the sake of simplicity, equation (3) is simplified to equation (4) allowing a numerical finite differences integration (the error on the final result has be checked to be less than $0.2 \%$ ).

$d F=2^{n} a F^{n}\left(1+\frac{d F}{2 F}\right)^{n} \frac{\sin \frac{n d \theta}{2}}{\cos \frac{d \theta}{2}}$

$d F=2^{n} a F^{n} \frac{\sin \frac{n d \theta}{2}}{\cos \frac{d \theta}{2}}$

This improved drive belt formula simulates the capstan test and accounts for the normal load dependence. For each sliding velocity, the experimental data recorded during ascent and descent steps were analysed to identify the values of parameters $a$ and $n$. This improved analysis shows that the dependence of friction to the normal load describes perfectly the change of $F_{1}$ for both ascent and descent of the crosshead irrespective of the dead weight $F_{0}$. Indeed, the average normal load is a function of both tensions at each strand of the yarn, $F_{0}$ and $F_{1}$, and is thus higher during the ascent of the crosshead since $F_{1}$ is higher than during the descent while $F_{0}$ remains the same. Moreover the parameter $n$ does not change significantly with the sliding velocity and an average value was considered. For E-Glass fibres, the parameter $a$ proved to be sensitive to the sliding velocity and the experimental data are perfectly fitted by a power law $\left(a=b V^{p}\right)$. Finally, the coefficient of kinetic friction was supposed to be a function of the normal load and the sliding velocity, equation (5).

$\mu_{k}=b V^{p} F_{n}^{n-1}$

Table 3

Parameters of equation (5) identified for E-glass and basalt fibres.

\begin{tabular}{llll}
\hline & $b\left(\mathrm{~s}^{\mathrm{p}} \mathrm{mm}^{-\mathrm{p}} \mathrm{N}^{-\mathrm{n}+1}\right)$ & $p$ & $n$ \\
\hline E-Glass yarn & 0.159 & 0.0246 & 0.948 \\
Basalt yarn & 0.137 & 0 & 0.909 \\
\hline
\end{tabular}

The final values of each parameter for both E-Glass and basalt yarns have been identified by a Newton-Raphson optimisation technique and are reported in Table 3 . To validate the results of this identification, the experimental load $F_{1}$ measured for each testing condition was compared in Fig. 3 to the trend predicted through equations (4)-(5). Irrespective of the dead weight and sliding velocity, this comparison shows that the model perfectly fits the experimental data for both ascent and descent of the crosshead.

\section{Calculations}

The present study introduces friction in the plane model proposed by Hong (Hong et al., 2002) and used by some other authors (Dusserre et al., 2014; de Araújo et al., 2003; Abel et al., 2012). This model, based on the elastica theory, considers the yarn as a curved cylindrical elastic homogeneous rod with a loop shape. The description of the model in this section uses the same notations as in the original model (Hong et al., 2002). The yarn is assumed to behave as a Euler-Bernoulli beam, its bending stiffness, $B$, is assumed to be constant and uniform and its value is calculated as the sum of the bending stiffness of each filament. This is obviously a very strong assumption with regard to the actual bending behaviour of a yarn (Syerko et al., 2012; Cornelissen and Akkerman, 2009), but the curvature change is not so large during stretching because the initial state considered is not the straight yarn, allowing a linearization of the behaviour.

\subsection{Geometrical relations}

The model considers a first set of four equations obtained from geometrical considerations, equation (6). These equations are exactly the same as in the original model.

$\left\{\begin{array}{l}\frac{W}{4}=X_{B}-\frac{d}{2} \cos \left(\beta-\frac{\pi}{2}\right) \\ \frac{C}{2}=Y_{B}-\frac{d}{2} \sin \left(\beta-\frac{\pi}{2}\right) \\ X_{B}-X_{A}=\frac{d}{2} \cos \left(\beta-\frac{\pi}{2}\right) \\ \frac{L}{4}=s_{A B}+s_{B C}\end{array}\right.$

\subsection{Momentum balance}

\subsubsection{Momentum balance of a quarter stitch}

The static equilibrium of the quarter stitch leads to a set of equations that relates the horizontal force $T$ and the moment $M$ acting at point $C$, and the reaction force $R$ acting at point $B$ to the force $P$ acting at point $A$. The set of equations (7) expresses the different loads acting on the quarter stitch as functions of the forces $R$ and $P$ and of the angles $\beta, \gamma$ and $\phi$. Here, the friction is not neglected and the force $R$ is not assumed perpendicular to the yarn at point $B$. The friction angle $\phi$ is introduced (Fig. 4) and will be used to account for friction in the model, cf. Section 3.3. It is noteworthy that the problem with friction can be formulated exactly in the same way as the problem without friction, except the parameter $k_{1}^{\prime}$, which includes the friction angle and replaces the parameter $k_{1}$ of the original model. 


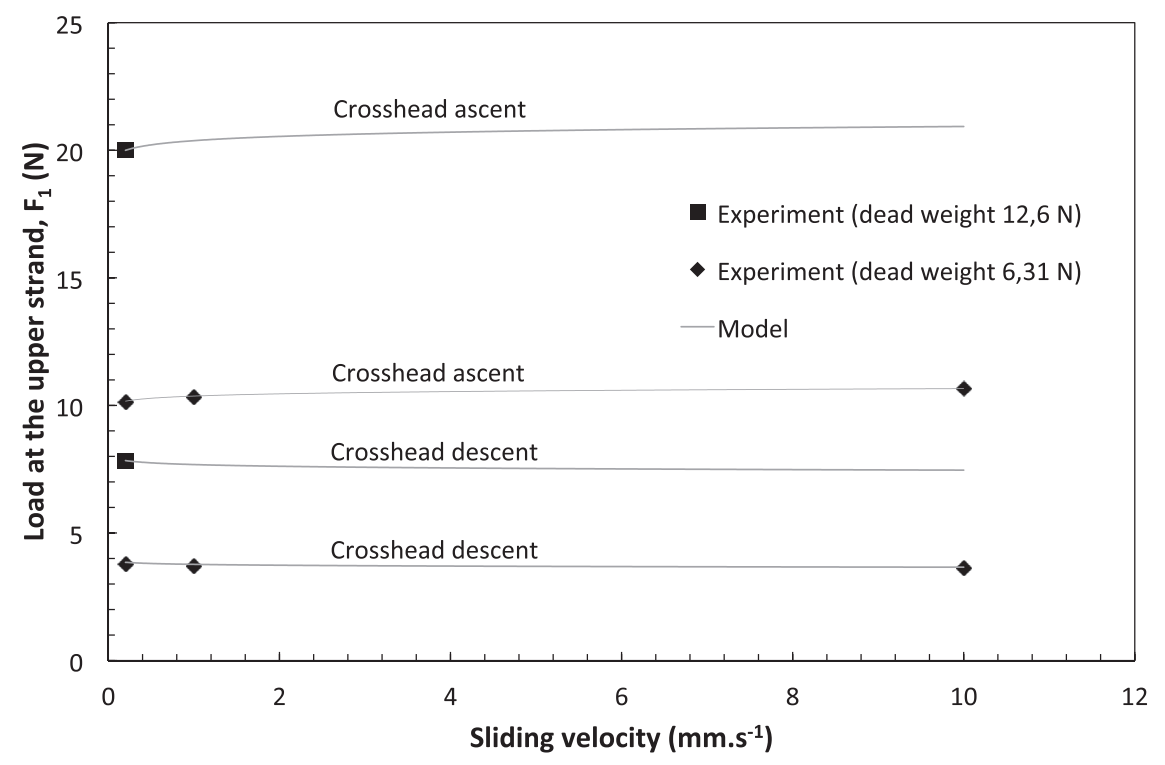

a.

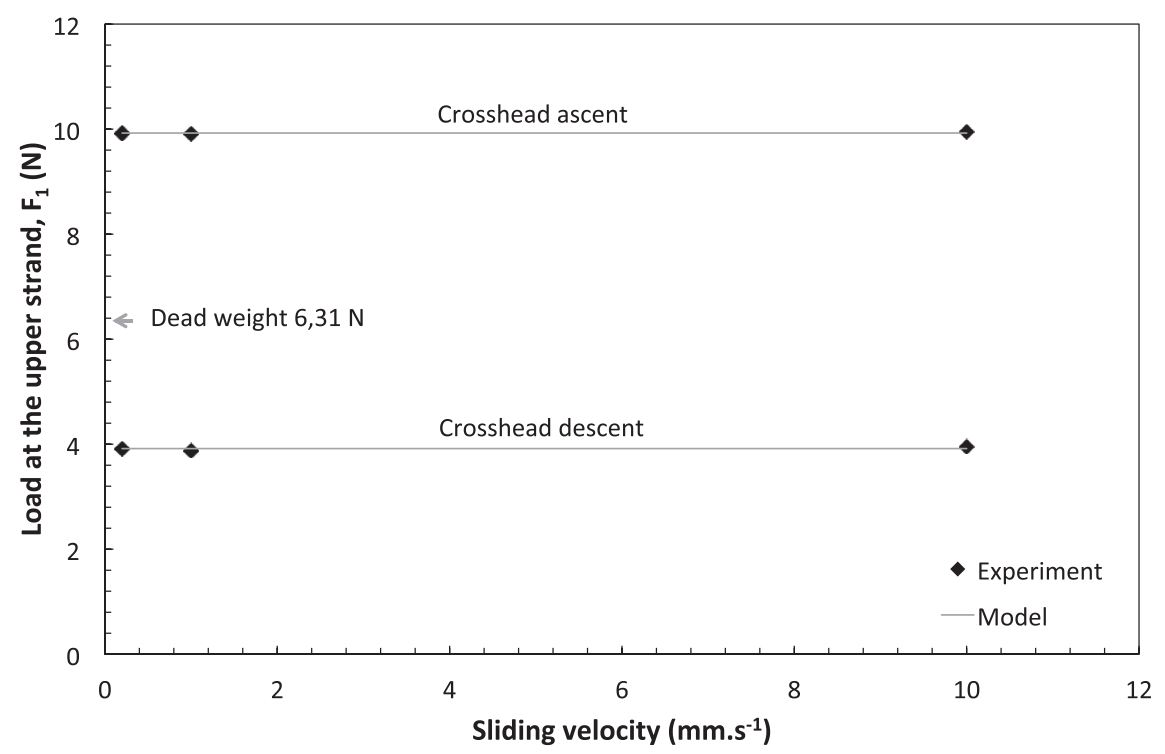

b.

Fig. 3. Comparison of the values of the load $F_{1}$ measured and simulated with equations (4) and (5): (a) E-glass yarns and (b) basalt yarns.

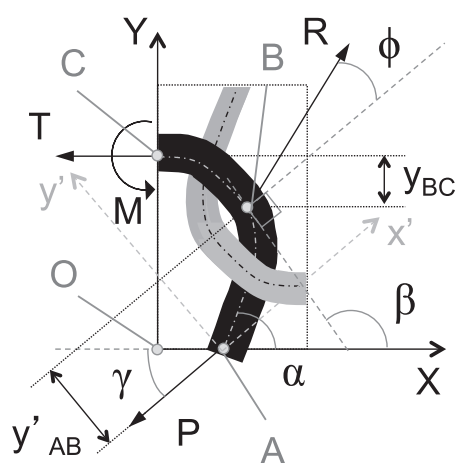

Fig. 4. Schematic view of the loads acting on a quarter loop.

$$
\left\{\begin{array}{l}
R_{X}=R \cos \left(\beta-\frac{\pi}{2}+\phi\right) \\
R_{Y}=R \sin \left(\beta-\frac{\pi}{2}+\phi\right)=P \sin \gamma \\
T=-P k_{1}^{\prime} \\
M=P\left(y_{A B}^{\prime}+k_{1}^{\prime} y_{B C}\right) \\
k_{1}^{\prime}=\cos \gamma+\sin \gamma \cdot \tan (\beta+\phi) \\
k_{1}=\cos \gamma+\sin \gamma \cdot \tan \beta
\end{array}\right.
$$

3.2.2. Momentum balance at an arbitrary point

The static equilibrium of a yarn segment between point $A$ and an arbitrary point of segment $A B$ leads to a set of differential equations that gives the values of the coordinate of the considered arbitrary 
point as well as the yarn length up to this point. This set of equations is written in its integral form in equation (8) and provides the expression of the coordinates of vector $A B$ as well as the yarn length between $A$ and $B$.

$$
\left\{\begin{array}{l}
s_{A B}=\frac{1}{2} \sqrt{\frac{B}{P}} \int_{\alpha-\gamma}^{\beta-\gamma} \frac{d \theta^{\prime}}{\left[\cos ^{2}\left(\frac{\alpha-\gamma}{2}\right)-\cos ^{2}\left(\frac{\theta^{\prime}}{2}\right)\right]^{1 / 2}} \\
x_{A B}^{\prime}=\frac{1}{2} \sqrt{\frac{B}{P}} \int_{\alpha-\gamma}^{\beta-\gamma} \frac{\cos \theta^{\prime} d \theta^{\prime}}{\left[\cos ^{2}\left(\frac{\alpha-\gamma}{2}\right)-\cos ^{2}\left(\frac{\theta^{\prime}}{2}\right)\right]^{1 / 2}} \\
y_{A B}^{\prime}=\frac{1}{2} \sqrt{\frac{B}{P}} \int_{\alpha-\gamma}^{\beta-\gamma} \frac{\sin \theta^{\prime} d \theta^{\prime}}{\left[\cos ^{2}\left(\frac{\alpha-\gamma}{2}\right)-\cos ^{2}\left(\frac{\theta^{\prime}}{2}\right)\right]^{1 / 2}} \\
X_{B}-X_{A}=x_{A B}^{\prime} \cos \gamma-y_{A B}^{\prime} \sin \gamma \\
Y_{B}=x_{A B}^{\prime} \sin \gamma+y_{A B}^{\prime} \cos \gamma
\end{array}\right.
$$

A similar analysis performed on the segment $B C$ leads to the set of equations (9) that provides the coordinates of vector $B C$ as well as the yarn length between $B$ and $C$. It is noteworthy that equations (8) and (9) are usually written under the form of complete and incomplete elliptic integrals of the first and second kinds thanks to variable changes. However these variable changes require considering different cases to account for the sign of the original variable. It was thus preferred to keep the integrals under the aforementioned forms that can be computed as easily as the elliptic integrals thanks to the trapezoidal rule.

$$
\left\{\begin{array}{l}
\varepsilon_{1}=\cos \left(\frac{\alpha-\gamma}{2}\right) \\
\varphi_{1 B}=\arcsin \left(\frac{\cos \left(\frac{\beta-\gamma}{2}\right)}{\varepsilon_{1}}\right) \\
s_{B C}=\sqrt{\frac{B}{2 P}} \int_{0}^{\pi-\beta} \frac{d \theta}{\left(k_{1} \cos \beta+2 \varepsilon_{1}^{2} \cos ^{2} \varphi_{1 B}+k_{1} \cos \theta\right)^{1 / 2}} \\
X_{B}=\sqrt{\frac{B}{2 P}} \int_{0}^{\pi-\beta} \frac{\cos ^{1 / \beta} d \theta}{\left(k_{1} \cos \beta+2 \varepsilon_{1}^{2} \cos ^{2} \varphi_{1 B}+k_{1} \cos \theta\right)^{1 / 2}}
\end{array}\right.
$$

\subsection{Resolution}

\subsubsection{Initial state}

The model detailed above allows to compute the values of all loads acting on the quarter loop, as well as the loop shape, if the four angles $\alpha, \beta, \gamma$ and $\phi$ are known. Solving the model requires a first initialisation state to compute the initial shape of the quarter loop. The angles $\alpha, \beta$ and $\gamma$ are not easily accessed through experimental observations and it is preferred to find their initial values thanks to an inverse method to match the average dimensions of a loop. Indeed the average yarn length, course length and wale width are standard characteristics of a knit. These data are sufficient in the initial model, but due to the introduction of friction, another datum is mandatory to initialize the model. It is here proposed to arbitrarily set the friction angle at a value comprised between $-\phi_{\max }$ and $\phi_{\max }$. This choice was motivated by the difficulty to measure a relevant data sensitive to the friction angle. The effect of the friction angle initial value on the results of the model will be discussed further. The optimization reported in this paper was performed with the Matlab ${ }^{\mathbb{B}}$ function fminsearch based on the Nelder-Mead simplex derivative-free method (Nelder and Mead, 1965).

\subsubsection{Successive steps of a stretching cycle}

Once the model initialized, a quasi-static tensile cycle can be simulated as successive equilibrium states. For each equilibrium state, the inverse method is solved and imposes the relevant data for each step of the cycle. The main issue to obtain relevant results remains the choice of the four parameters whose value must be imposed during each step of the cycle. The parameters chosen for each step of the cycle in this study are compiled in Table 4 and Fig. 5 depicts an example of the model results showing the different steps and the corresponding behaviour considering the assumption explained further. A first linear part corresponds to the adherence, followed by a second linear part with a much smaller slope during sliding, up to jamming characterized by an exponential curve. The unloading is similar to the loading but with a higher slope of the exponential curve leading to a pronounced hysteretic behaviour before sliding. Finally a linear part with a low slope describes the sliding step up to a state at rest characterized by an irreversible deformation.

The first parameter imposed is the yarn length per stitch, $L$, whose value is known and constant all along the cycle since the yarn is assumed to be inextensible. The value of the stitch width, $W$, is known for the knit at rest and can be set to the value $4 d$ during the jamming steps. The value of the yarn length between the points $B$ and $C$ can be kept constant during the static friction steps. One of the parameter of the problem must be incremented at each time to impose a wale-wise stretching. This is achieved with the parameters $C$ and $\phi$. The initial value of the course height, $C_{0}$, is known and its value can be incremented during the sliding and jamming steps, as in the original model. The initial value of the angle $\phi$ is taken arbitrarily, and the parameter can be incremented during the static friction steps up to its maximal or minimal value respectively during the loading and unloading steps. During the sliding steps, the value of $\phi$ is kept constant.

For most of the steps, the fourth parameter to impose is chosen more arbitrarily. Some experimental observation suggested that the angle $\beta$ remains constant during the static friction step of the

Table 4

\begin{tabular}{|c|c|c|c|c|c|c|c|c|c|c|}
\hline \multirow[t]{2}{*}{ Step } & & & \multicolumn{8}{|c|}{ Parameters } \\
\hline & & & $\beta$ & $s_{B C}$ & $T$ & $R n$ & C & W & $L$ & $\phi$ \\
\hline Initialisation & $A$ & SF & & & & & $C_{0}$ & $W_{0}$ & $L_{0}$ & $\phi_{0}$ \\
\hline \multirow[t]{3}{*}{ Loading } & $A-B$ & $\mathrm{SF}$ & & $S_{B C_{-} A}$ & & $R_{n \_A}$ & & & $L_{0}$ & 2 \\
\hline & $B-C$ & $\mathrm{~S}$ & & & & $R_{n \_B}$ & $\pi$ & & $L_{0}$ & $\phi_{\max }$ \\
\hline & $C-D$ & $\mathrm{~J}$ & & & & & $\pi$ & $4 d$ & $L_{0}$ & $\phi_{\max }$ \\
\hline \multirow[t]{2}{*}{ Unloading } & $D-E$ & SF & $\beta_{-} D$ & $S_{B C_{-} D}$ & & & & & $L_{0}$ & $y$ \\
\hline & $E-F$ & $S$ & & & $T_{-E}$ & & $y$ & & $L_{0}$ & $-\phi_{\max }$ \\
\hline \multirow[t]{3}{*}{ Loading } & $F-B^{\prime}$ & $\mathrm{SF}$ & & $S_{B C \_F}$ & & $R_{n \_F}$ & & & $L_{0}$ & $\pi$ \\
\hline & $B^{\prime}-C^{\prime}$ & $\mathrm{S}$ & & & & $R_{n \_B^{\prime}}$ & 7 & & $L_{0}$ & $\phi_{\max }$ \\
\hline & $C^{\prime}-D^{\prime}$ & $\mathrm{J}$ & & & & & $\pi$ & $4 d$ & $L_{0}$ & $\phi_{\max }$ \\
\hline
\end{tabular}

Parameters imposed during each step of a tensile cycle.

SF: static friction; S: sliding; J: jamming. 


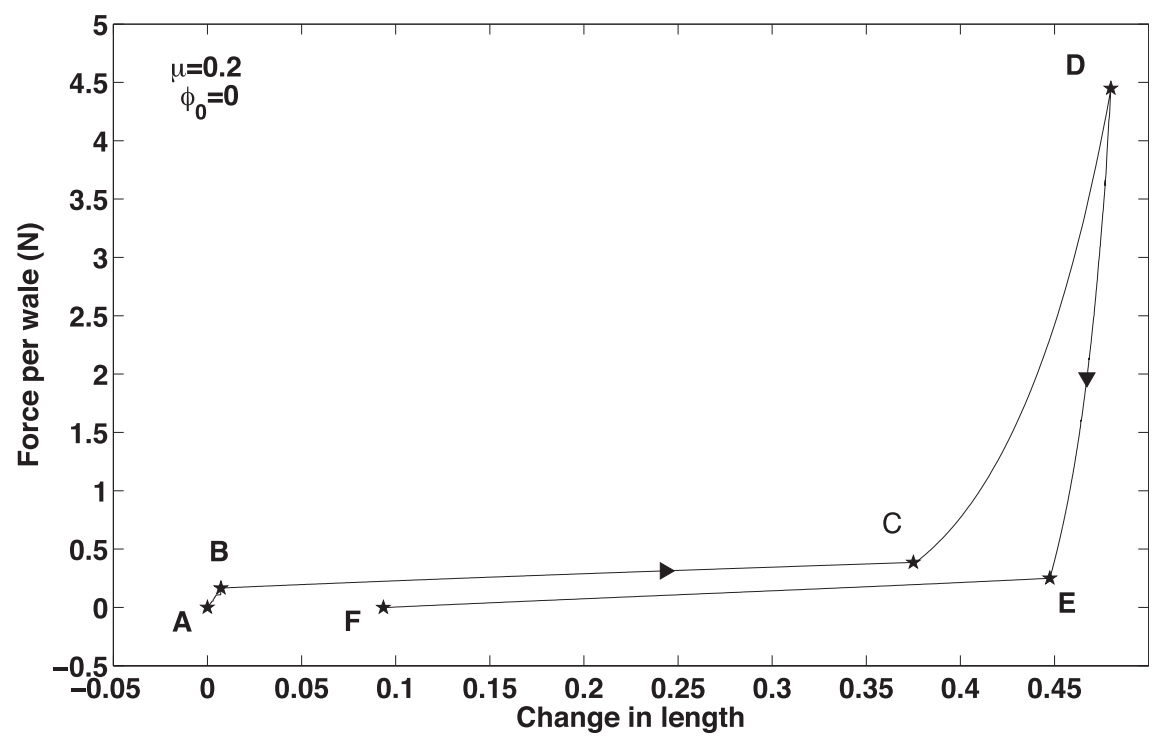

Fig. 5. Computed tensile behaviour during a loading-unloading cycle.

unloading. Despite a lack of physical interpretation, this assumption proved to provide relevant results whereas the other parameters tested were not satisfactory. During the sliding steps it was first assumed that the tangential component of the reaction load at point $B$ could be considered as constant. Moreover, during these steps, the angle $\phi$ is also constant. Thus the normal component of $R$ is also constant. As it didn't make sense to impose the tangential component of $R$ during the static friction, where the friction contribution increases, it was chosen to impose its normal component, $R_{n}$, during both static friction and sliding steps of the loading. This assumption was not considered during the unloading because it was not compatible with the choice of $\beta$ for the static friction step. It was thus arbitrarily chosen to consider the original assumption of Hong (Hong et al., 2002) that proposed to keep constant the force $T$ at point $C$.

It is noteworthy that some of these assumptions include an arbitrary part. There is maybe no physical reason to assume that a parameter remains constant during one of these steps. However they provide the most physically reasonable results and it was not the scope of the paper to understand in detail their physical sense.

\subsection{Apparent coefficient of kinetic friction}

The aforementioned model accounts for friction by introducing a constant ratio, $\mu$, between the normal and tangential component of the reaction force $R$ at point $B$, through the angle $\phi(\mu=\tan \phi)$. It was shown in the previous section that the value of the coefficient of kinetic friction depends on the value of the normal contact load and also on the sliding velocity for E-glass fibres. However these data are not well known and change along with the stretching of the knit.

For the sake of simplicity, the previous model was implemented only to account for a constant coefficient of kinetic friction, corresponding to average values of normal load and sliding velocity. The average values were assessed from the analytical results obtained by Balea (2011) using the original model without friction. During the first step of the stretching, the sliding velocity at the loop crossing point $B$ is about $0.5 \mathrm{~mm} \mathrm{~s}^{-1}$ for a crosshead displacement rate of $50 \mathrm{~mm} \mathrm{~min}^{-1}$, and the normal load is about $1 \mathrm{~N}$. According to equation (5) and Table 3, the average value of the coefficient of kinetic friction $\mu_{k}$ will be set to 0.156 for the contact between Eglass yarns and a value of 0.137 for the contact between basalt yarns.

Furthermore the contact between both quarter stitches can be assimilated to a yarn coiled perpendicularly around the other yarn according to an angle $\theta$. Thus, even for a constant coefficient of static friction, the drive belt formula, equation (1), implies that the ratio between normal and tangential contact loads, $\mu$, also depends on the coiling angle, $\theta$ (equation (10)). Obviously this angle also changes during the stretching of the knit. The minimal value of this angle was assessed from experimental observations of the unloaded knit (Fig. 6a and b). The maximal value of $\theta$ was assessed through a plane geometrical model of the jammed knit (Fig. 6c and equation (11)). For E-glass knits, the coiling angle $\theta$ ranges between $72^{\circ}$ and $149^{\circ}$, whereas it ranges between $66^{\circ}$ and $147^{\circ}$ for basalt knits. This implies a value of $\mu$ ranging between 0.06 and 0.6 from the beginning of the stretching test to the jamming point. It is noteworthy that this effect is much more important than those of sliding velocity and normal load. In the following section, the ratio $\mu$ will be assumed to be constant and set to the value corresponding to the average coiling angle, respectively $110^{\circ}$ and $106^{\circ}$ for E-glass and basalt knits, i.e. respectively $\mu=0.20$ and $\mu=0.17$.

$\mu=\frac{R_{t}}{R_{n}}=\frac{e^{\mu_{k} \theta}-1}{e^{\mu_{k} \theta}+1} \tan \frac{\theta}{2}$

$$
\frac{L}{4}=\frac{d}{2}+\theta d+\frac{\frac{c}{2}-d \sin \left(\theta-\frac{\pi}{2}\right)}{\cos \left(\theta-\frac{\pi}{2}\right)}
$$

\section{Results and discussion}

\subsection{Monotonic stretching}

The model detailed in the previous section was first used to perform a sensitivity study on the effect of friction on the monotonic stretching behaviour of a plain-knit in the wale-wise direction. 

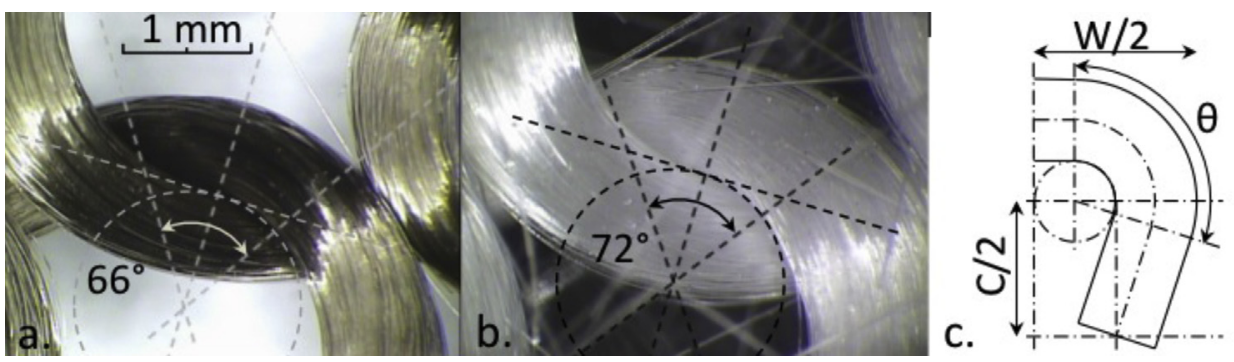

Fig. 6. Assessment of the minimal coiling angle from unloaded knit observation of (a) basalt and (b) E-glass knits, and maximal coiling angle (c) from geometrical modelling.

\subsubsection{Effect of the coefficient of kinetic friction}

Fig. 7 displays the results provided by the model for different values of the coefficient of kinetic friction, assuming that the initial state corresponds to the sliding limit $\left(\phi_{0}=\phi_{\max }\right)$. Fig. 7a shows that the behaviour is linear before jamming and that the value of the coefficient of friction mainly affects the exponential behaviour after jamming. Indeed, the force per wale computed at a change in length of 0.45 with a coefficient of kinetic friction of 0.2 is $20 \%$

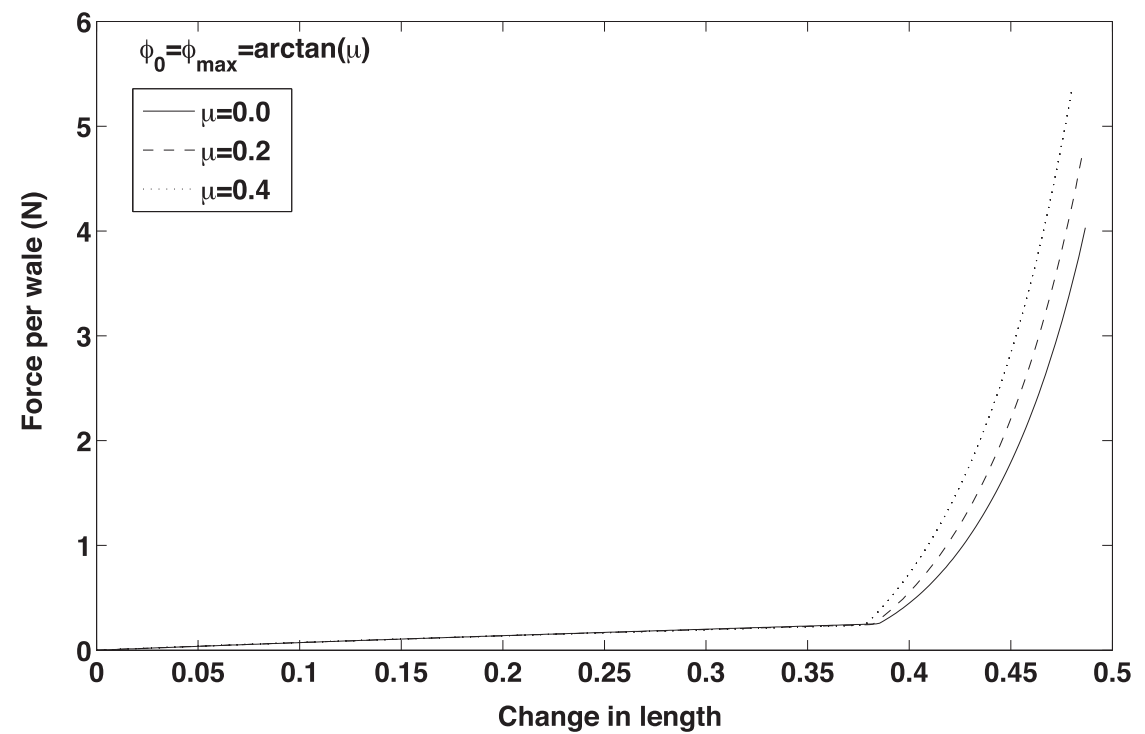

a.

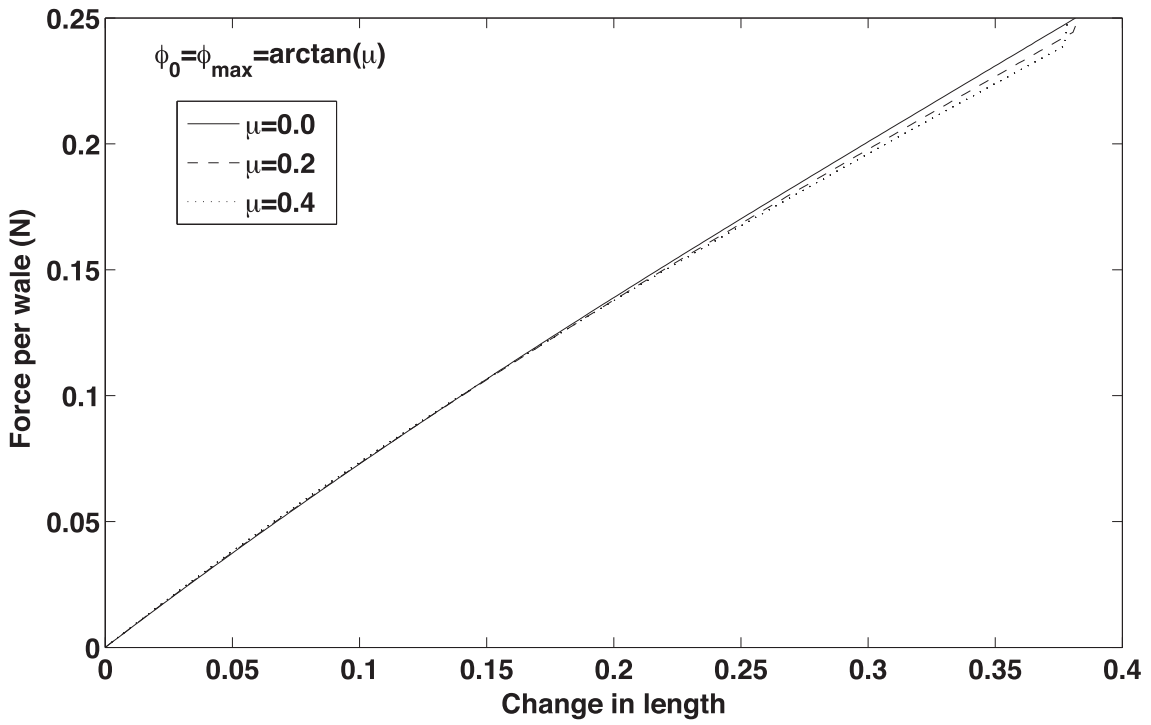

b.

Fig. 7. Effect of the coefficient of friction on the stretching behaviour for $\phi_{0}=\phi_{\max }$ : (a) full-scale behaviour (b) behaviour before jamming. 


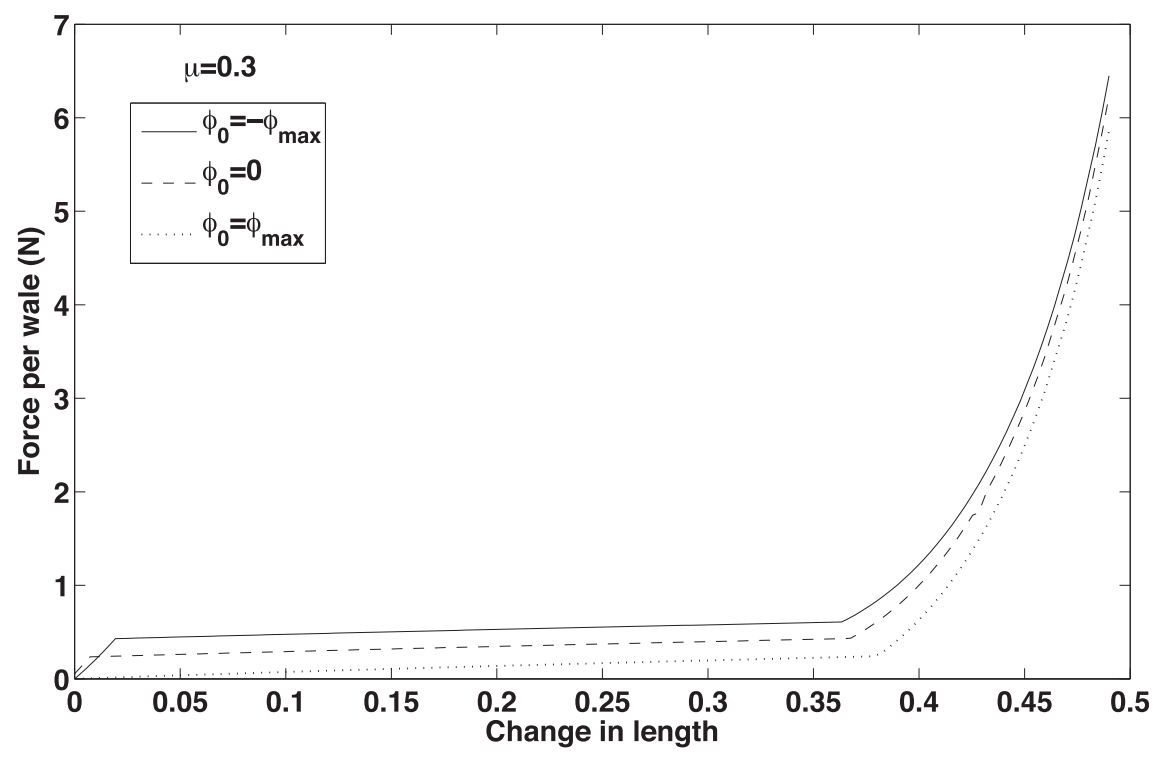

Fig. 8. Effect of the initial value of angle $\phi$ on the stretching behaviour at loading for $\mu=0.3$.

higher than without friction. With a coefficient of friction of 0.4 , this value is $60 \%$ higher than without friction.

Fig. 7b details the stretching behaviour before jamming. It is noteworthy that this part of the behaviour is not perfectly linear and that friction increases this non-linearity. Surprisingly friction tends to slightly decrease the stiffness before jamming: at jamming, the force per wale is $4 \%$ lower with a coefficient of friction of 0.4 than without friction. Moreover jamming occurs slightly earlier with friction. These both statements are explained by the tangential component introduced at the loops crossing point that contributes to stretch the loop more easily.

\subsubsection{Effect of the initial friction angle}

Fig. 8 displays the analytical results obtained with an apparent coefficient of friction of 0.3 for different initial values of the angle $\phi$. The value $\phi_{0}=\phi_{\max }=\arctan (\mu)$ corresponds to an initial state at the limit of sliding in the stretching direction, the value $\phi_{0}=0$ corresponds to a force $R$ perpendicular to the yarn in the initial state, and the value $\phi_{0}=-\phi_{\max }$ corresponds to an initial state at the limit of sliding in the direction opposite to the stretching direction.

At the beginning of the stretching, before sliding, a first linear part arises and the slope is similar irrespective of the initial value of $\phi$. Then sliding occurs at a change in length obviously function of $\phi_{0}$. The almost linear part corresponding to the sliding step is not affected by the initial value of $\phi$, except its duration. The higher the initial value of $\phi$ is, the higher is the change in length at jamming. This is consistent with the more stretched state at the beginning of sliding with a low value of $\phi_{0}$. Indeed a shorter sliding distance will be necessary to rich the limit width value.

After jamming, the exponential curve is almost only shifted vertically and all behaviours seem to tend toward the same curve.

\subsubsection{Effect of the fibre type}

Fig. 9 compares the experimental monotonic stretching behaviour of both E-glass and basalt knits in the wale-wise direction to the results computed using the data of Table 1 and the average apparent coefficient of kinetic friction assessed in the previous section. On the overall view of Fig. 9a, the behaviour at the beginning of stretching seems to be properly described, but afterwards the curve remains almost linear up to jamming contrarily to the actual knit behaviour. This trend may be due to the simplified assumption of the model. Indeed the ratio $\mu$ is kept constant whereas it was proved experimentally that it might strongly vary during the test as function of normal load, sliding velocity and overall coiling angle. Moreover, the yarn diameter is also kept constant neglecting the yarn transverse compaction that would change the jamming point as well as the yarn bending stiffness. Fig. 9b shows that introducing friction in the model accounts for a phenomenon that was not described in the original model. Indeed the initial slope curve is higher than during sliding. The improved model reproduces qualitatively this slope change. However the modelled behaviour is not smooth enough due to the aforementioned assumptions. Adding a sensitivity of the coefficient of kinetic friction to the normal load would help to better model this part of the curve.

\subsection{Cyclic stretching}

The results displayed in Fig. 10 show that the wale-wise stretching behaviour of E-glass plain knit under study is highly hysteretic, with a slope almost vertical at the beginning of the unloading step, and a residual strain of $15 \%$ after the first cycle. The second step is similar to the first one, but the maximal load is only of $4 \mathrm{~N}$ per wale instead of $5 \mathrm{~N}$ per wale at the end of the first cycle. The third cycle is very similar to the second one despite a slight increase of the residual strain and a slight decrease of the maximal load.

Fig. 11 shows the hysteretic behaviour obtained by simulating a cyclic test with the model enriched by friction. This curve will be compared qualitatively to the experimental curve of Fig. 10. The improved model properly describes the beginning of the unloading step even if the curve is not vertical as it is the case experimentally. Afterwards, the transition between the adherence and the sliding step leads to a slope discontinuity whereas the experimental curve is smoother. Obviously the sliding step of unloading is almost linear and parallel to the sliding curve of loading. The force per stitch decreases until it reaches zero. The second cycle then begins. The first step, describing adherence between both crossing loops, consists to increase the value of $\phi$ from its value at the end of the first cycle $\left(-\phi_{\max }\right.$ during the sliding step of unloading) up to its value during the sliding step of loading, $\phi_{\max }$. Due to the initial value 


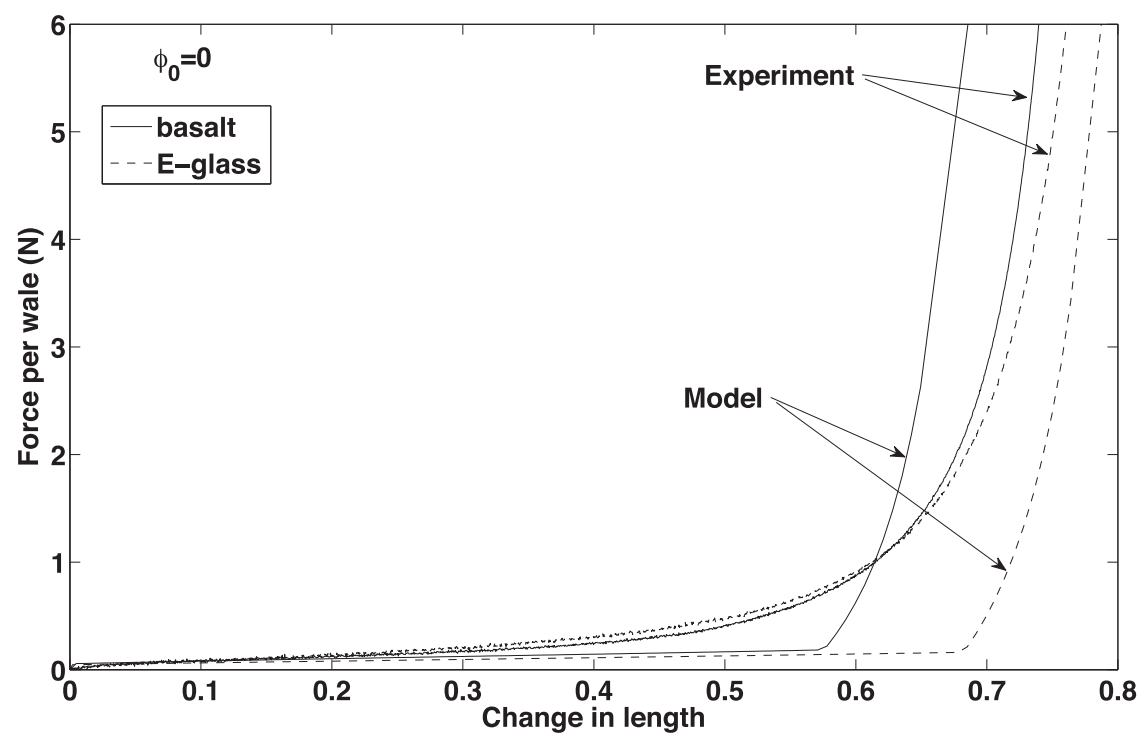

a.

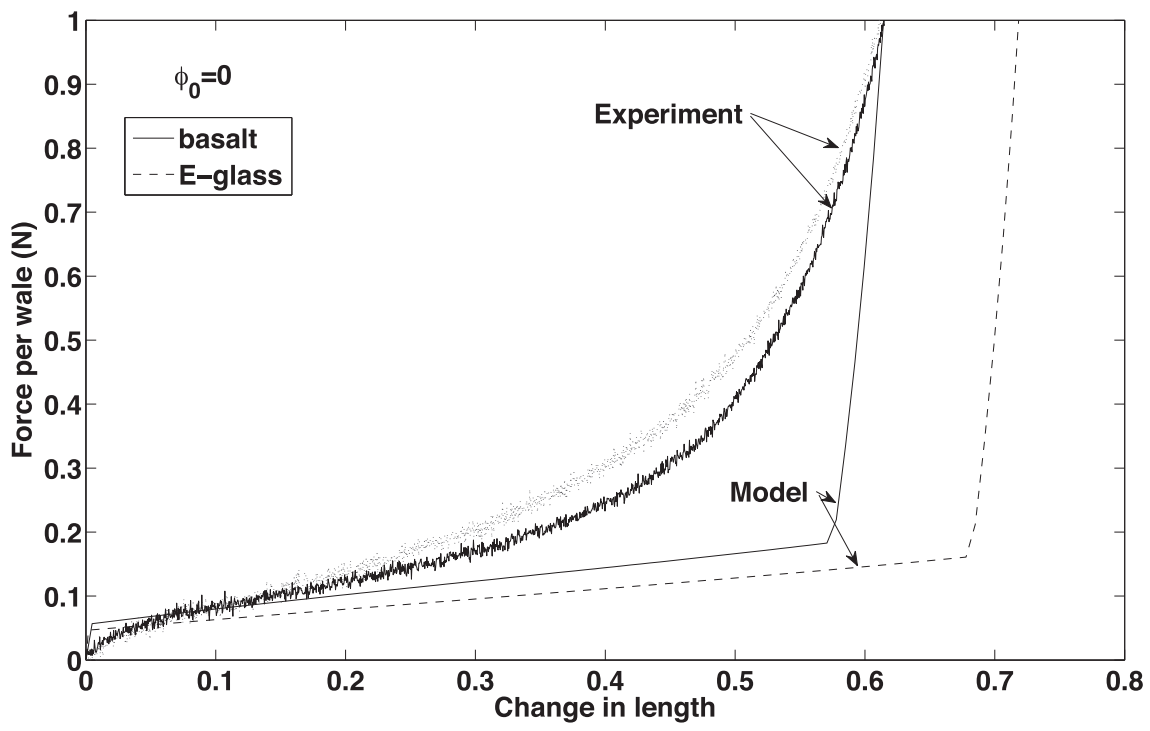

b.

Fig. 9. Comparison of experimental and computed wale-wise stretching behaviours of E-glass and basalt knits.

chosen $\phi_{0}=0$, and to the low slope of the curve during the sliding steps, the load is higher during the second step than during the first one. This is not the case experimentally and it may indicate that the initial value of $\phi$ is maybe close to $-\phi_{\max }$. It could be explained by the knit stretching during knitting. Indeed the angle $\phi$ in the knit at rest would be at the sliding limit in the unloading direction. However it probably varies significantly during the numerous handlings of the knit before testing.

\section{Conclusion}

This paper reports the results of an experimental and theoretical analysis of the effect of friction on the stretching behaviour of a technical plain knit.

A first experimental study showed that the yarn-to-yarn coefficient of kinetic friction between perpendicular E-glass fibres depends on both sliding velocity and normal load. Both effects can be perfectly described by power laws. A similar result is obtained with basalt fibres except that friction is not sensitive to the sliding velocity in the investigated range.

Then a theoretical analysis of the friction between two perpendicular yarns was performed to include its contribution in an existing semi-analytical model describing the wale-wise stretching behaviour of technical knits. This model was used to perform a sensitivity study that showed the effect of the coefficient of kinetic friction and of the initial friction angle on the stretching behaviour of the knit. The former only influences the behaviour after jamming of the knit whereas the latter mainly influences the behaviour before jamming. A comparison of the computed results with experimental data shows that introducing friction improves the ability of the model to describe the physical phenomena occurring during monotonic stretching, but the transition between the different steps is too sudden to reproduce the smooth experimental curves. 


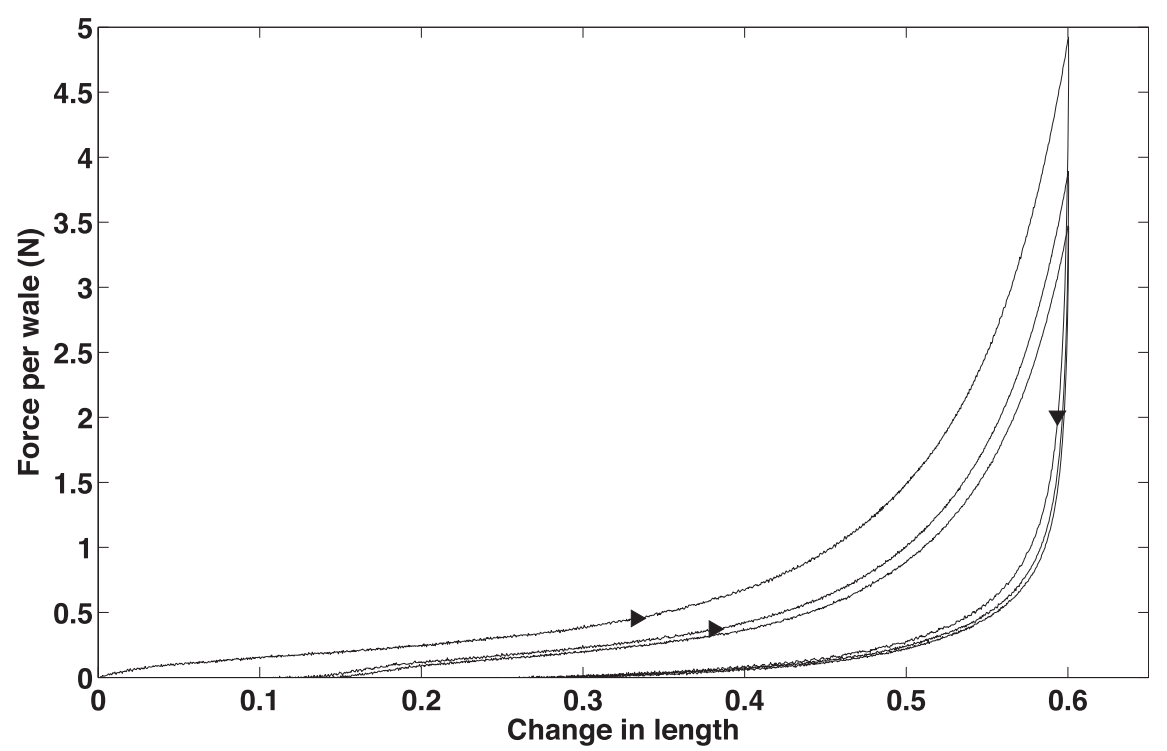

Fig. 10. Hysteretic wale-wise stretching behaviour of E-glass fibre plain knit.

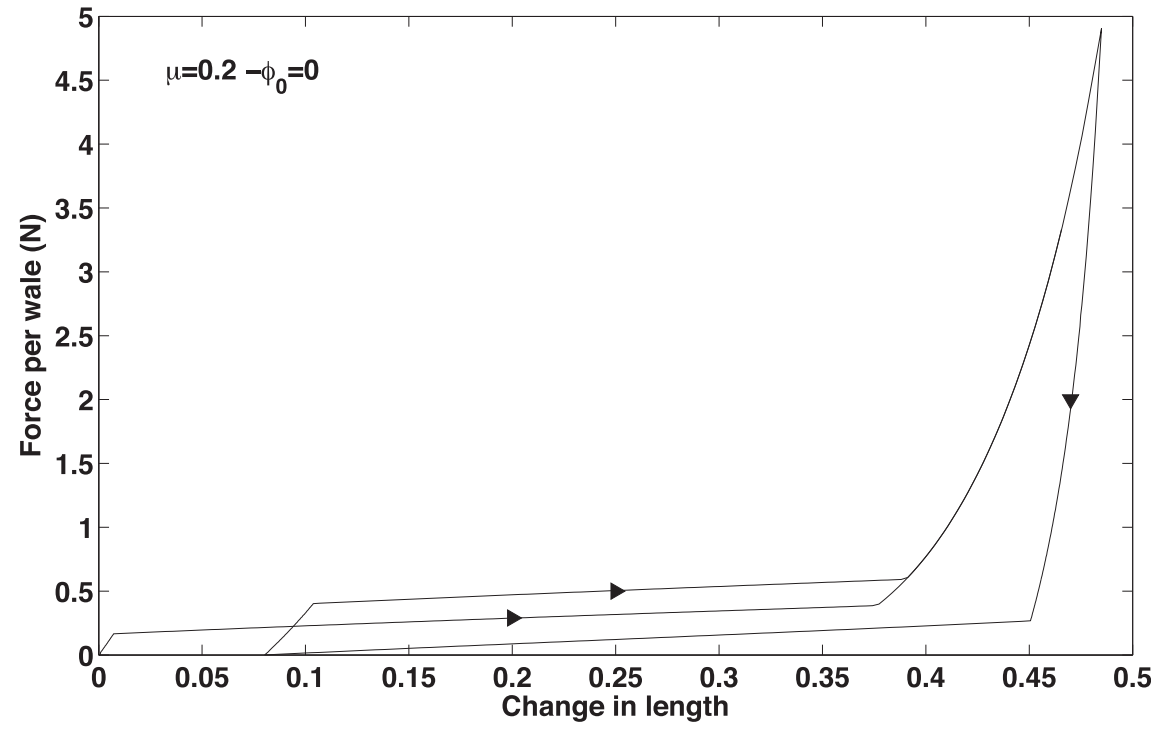

Fig. 11. Modelled hysteretic stretching behaviour during two consecutive loading-unloading cycles for $\phi_{0}=0$ and $\mu=0.2$.

A simulation of a cyclic stretching thanks to this model proved that sliding with friction at the loops crossing point is the main mechanism that involves the hysteretic behaviour of a plain knit in the wale-wise direction. However the modelled behaviour is not so smooth as the actual one. The sensitivity of friction to normal load and coiling angle may contribute to this difference as well as friction inside the yarn, yarn transverse compaction and shear stresses.

More accurate results could be obtained by introducing a yarn transverse deformation through a link between the yarn diameter and the contact load at point B, as proposed in Balea (2011) and Dusserre and Bernhart (2015). Coupling the yarn transverse compaction with the present friction model, possibly improved by accounting for the friction sensitivity to normal load, sliding velocity and coiling angle, may provide hysteretic stretching curves similar to the experimental ones.

\section{Acknowledgement}

The author gratefully acknowledges Textile Aéro Tarn for supplying the specimens. Special thanks are expressed to Laura Balea, Gérard Berhnart, Francis Planel, Nicolas Dumont and Bernard Cabanes.

\section{References}

Abel, J., Luntz, J., Brei, D., 2012. A two-dimensional analytical model and experimental validation of garter stitch knitted shape memory alloy actuator architecture. Smart Mater. Struct. 21, 085011.

Allaoui, S., Boisse, P., Chatel, S., et al., 2011. Experimental and numerical analyses of textile reinforcement forming of a tetrahedral shape. Compos. A Appl. Sci. Manuf. 42, 612-622.

Balea, L., 2011. Comportement des matériaux composites à renforts tricotés élaborés par injection de résine (Ph. D. thesis). University of Toulouse, France (in French). 
Balea, L., Dusserre, G., Bernhart, G., 2014. Mechanical behaviour of plain-knit reinforced injected composites: effect of inlay yarns and fibre type. Compos. B Eng. 56, 20-29.

Baltina, I., Cigle, E., Ozoliņa, N., 2007. The influence of sizing process to physical properties of glass yarn. Mater. Sci. Text Cloth. Technol. 2, 91-96.

Behary, N., Ghenaim, A., El Achari, A., et al., 2000. Tribological analysis of glass fibers using atomic force microscopy (AFM)/lateral force microscopy (LFM). J. Appl. Polym. Sci. 75, 1013-1025.

Blau, P.J., 2008. Friction Science and Technology: from Concepts to Applications, second ed. CRC Press, p. 67.

Cornelissen, B., Akkerman, R., 27 . Analysis of yarn bending behaviour. In: ICCM-17 17th International Conference on Composite Materials, Edinburgh, UK.

Cornelissen, B., Rietman, B., Akkerman, R., 2013. Frictional behaviour of high performance fibrous tows: friction experiments. Compos. A Appl. Sci. Manuf. 44, 95-104.

de Araújo, M., Fangueiro, R., Hong, H., 2003. Modelling and simulation of the mechanical behaviour of weft-knitted fabrics for technical applications. Part II: 3D model based on the elastica theory. Autex Res. J. 3, 166-172.

Duhovic, M., Bhattacharyya, D., 2006. Simulating the deformation mechanisms of knitted fabric composites. Compos. A Appl. Sci. Manuf. 37, 1897-1915.

Dusserre, G., Bernhart, G., 2015. Knitting processes for composites manufacture. In: Boisse, P., Jones, G. (Eds.), Advances in Composites Manufacturing and Process Design. Woodhead Publishing Ltd, ISBN 978-1-78242-307-2.

Dusserre, G., Jourdain, E., Bernhart, G., 2011. Effect of deformation on knitted glass preform in-plane permeability. Polym. Compos. 32, 18-28.

Dusserre, G., Balea, L., Bernhart, G., 2014. Elastic properties prediction of a knitted composite with inlaid yarns subjected to stretching: a coupled semi-analytical model. Compos. A Appl. Sci. Manuf. 64, 185-193.

Gupta, B.S., 1992. Frictional behaviour of fibrous materials. In: Fornes, R.E., Gilbert, R.D., Mark, H.E. (Eds.), Polymer and Fiber Science: Recent Advances. VCH Publisher, Inc., New-York, pp. 305-332.
Heardman, E., Lekakou, C., Bader, M.G., 2001. In-plane permeability of sheared fabrics. Compos. A Appl. Sci. Manuf. 32, 933-940.

Hong, $\mathrm{H}$, de Araujo, M.D. Fangueiro, $\mathrm{R}$, et al., 2002. Theoretical analysis of loadextension properties of plain weft knits made from high performance yarns for composite reinforcement. Text. Res. J. 72, 991-996.

Howell, H.G., Mazur, J., 1953. Amontons' law and fibre friction. J. Text. I Trans. 44, T59-T69.

Leong, K.H., Ramakrishna, S., Huang, Z.M., et al., 2000. The potential of knitting for engineering composites-a review. Compos. A Appl. Sci. Manuf. 31, $197-220$.

Matsuo, M., Yamada, T., 2009. Hysteresis of tensile load - strain route of knitted fabrics under extension and recovery processes estimated by strain history. Text. Res. J. 79, 275-284.

Nelder, J.A., Mead, R., 1965. A simplex method for function minimization. Comput J. 7, 308-313.

Padaki, N.V., Alagirusamy, R., Sugun, B.S., 2006. Knitted preforms for composite applications. J. Ind. Text. 35, 295-321.

Popper, P., 1966. The theoretical behavior of a knitted fabric subjected to biaxial stresses. Text. Res. J. 36, 148-157.

Senthilkumar, M., Anbumani, N., 2011. Dynamics of elastic knitted fabrics for sports wear. J. Ind. Text. 41, 13-24.

Syerko, E., Comas-Cardona, S., Binetruy, C., 2012. Models of mechanical properties/ behaviour of dry fibrous materials at various scales in bending and tension: a review. Compos. A Appl. Sci. Manuf. 43, 1365-1388.

Truong, T.C., Ivanov, D.S., Klimshin, D.V., et al., 2008. Carbon composites based on multi-axial multi-ply stitched preforms. Part 7: mechanical properties and damage observations in composites with sheared reinforcement. Compos. A Appl. Sci. Manuf. 39, 1380-1393. 